\title{
Morphologically Directed Raman Spectroscopy as a Novel Tool to Monitor Changes in Particle Size and Shape in Solid Dosage Blends and Formulations
}

\author{
C. L. LEVOGUER ${ }^{1}$, L. KIDDER ${ }^{2}$, K. HABER ${ }^{2}$ \\ ${ }^{1}$ Malvern Instruments Limited, Malvern, United Kingdom \\ ${ }^{2}$ Malvern Instruments Inc., Columbia MA, USA \\ E-mail: carl.levoguer@malvern.com (C. L. Levoguer)
}

Sci Pharm. 2010; 78: 713

doi:10.3797/scipharm.cespt.8.PPAT24

One of the key steps in the manufacturing of solid oral dosage formulations is the blending of several ingredients in powdered form which are then processed further to achieve the final dosage form. Up until this blending process, it is relatively straightforward to measure critical quality attributes such as particle size and/or shape of the input materials using well established techniques such as laser light scattering or particle image analysis. A much more difficult challenge is to be able to characterise the size and shape of the individual ingredients once they have been through the blending process. This may be especially important when trying to understand any possible impact of the blending process on the size and shape of the API and how this might impact the bioavailability or bioequivalence of the drug. The technique of NIR chemical imaging has been used to get some information of the homogeneity of powder blends [1], however limitations in spatial resolution impact its applicability for smaller particle sizes.

In this paper we describe a novel approach using a hybrid technique of Morphologically directed Raman Spectroscopy to study the effects of processing on a laboratory scale of a model formulation consisting of ibuprofen, starch and lactose. The sample is first of all dispersed in its dry state, and then images are captured of the individual particles in the mixture in order to determine their size and shape. Each particle is then targeted in an automated manner with a Raman microprobe to obtain a chemical ID. Particle size and shape distributions can then be constructed for each of the ingredients and compared directly with measurements made before the blending process. Results for the model formulation are presented and implications for a number of different types of pharmaceutical formulations are discussed.

[1] $\mathrm{Ma} \mathrm{H}$, Anderson CA, Characterization of pharmaceutical powder blends by NIR chemical imaging. J Pharm Sci. 2008; 97: 3305-3320. doi:10.1002/jps.21230 